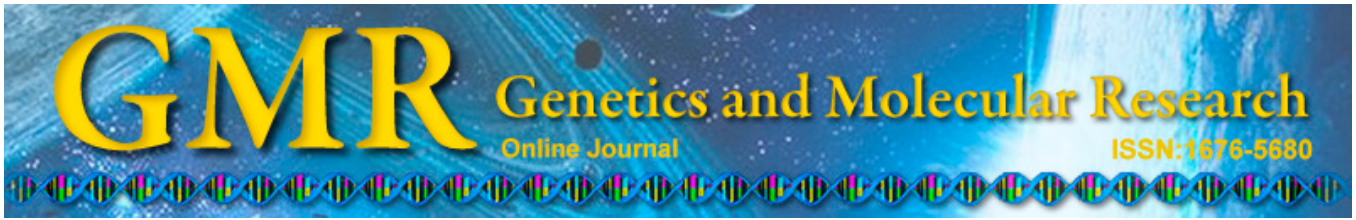

\title{
Bispectral index for monitoring anesthetic depth in patients with severe burns receiving target-controlled infusion of remifentanil and propofol
}

\author{
Z.G. Guo*, X.P. Jia*, X.Y. Wang, P. Li, X.J. Su and J.H. Hao \\ Department of Anesthesiology, \\ First Affiliated Hospital of General Hospital of PLA, Beijing, China \\ *These authors contributed equally to this study. \\ Corresponding author: J.H. Hao \\ E-mail: jiahuahaocn@126.com
}

Genet. Mol. Res. 14 (3): 7597-7604 (2015)

Received July 7, 2014

Accepted March 12, 2015

Published July 13, 2015

DOI http://dx.doi.org/10.4238/2015.July.13.3

\begin{abstract}
This study evaluated the feasibility and effectiveness of using the bispectral index (BIS) to monitor anesthetic depth in patients with severe burns receiving intravenous target-controlled infusion (TCI) of remifentanil and propofol. We randomly assigned 80 patients undergoing elective escharectomy ( $<1$ week) to BIS (A) and control (B) groups. All patients received remifentanil and propofol as intravenous TCI anesthesia. Clinical data were recorded at different time points. The time from drug withdrawal to eye opening upon the patient hearing his/ her name called and upon reaching an Aldrete score of 9 points was also recorded. During anesthesia maintenance, the target concentrations of remifentanil and propofol in group A were significantly lower than that in group B $(2.12 \pm 0.35 v s 2.50 \pm 0.21 \mathrm{ng} / \mathrm{mL}$ and $2.54 \pm 0.22$ vs $2.86 \pm$ $0.31 \mu \mathrm{g} / \mathrm{mL}$, respectively; $\mathrm{P}<0.01)$. The time from drug withdrawal to eye opening upon the patient hearing his/her name called and reaching an Aldrete score of 9 points in group A was considerably shorter than
\end{abstract}


that in group B $(7.90 \pm 0.58$ vs $8.35 \pm 0.66 \mathrm{~min}$ and $9.15 \pm 0.69$ vs 11.13 \pm 0.96 min, respectively; $\mathrm{P}<0.01$ ). In both groups, mean arterial pressure and heart rate values at each time point after loss of consciousness were significantly lower than the baseline values $(\mathrm{P}<0.05)$, with the exception of $2 \mathrm{~min}$ after intubation. The use of BIS to monitor anesthetic depth in patients with severe burns receiving TCI of remifentanil and propofol during the perioperative period reduces propofol consumption and shortens the consciousness recovery time in patients.

Key words: Propofol; Remifentanil; Target-control infusion; Burn

\section{INTRODUCTION}

Post-burn pathophysiological responses can lead to changes in pharmacokinetic parameters, and the extent of these pharmacokinetic changes is dependent on the severity and course of the burn. After the acute phase of a severe burn, the blood flow volumes in the liver and kidneys increase with the presence of hypermetabolism and increasing cardiac output, which leads to enhanced drug clearance. The recovery of hepatic and renal functions varies according to the individual (Han et al., 2009; Kaneda and Han, 2009; Yamashita et al., 2010; Brunette et al., 2011). The combination of remifentanil and propofol for intravenous targetcontrolled infusion (TCI) has been extensively used for various types of surgical anesthesia. Numerous studies have proven that intravenous TCI enables more precise control over intravenous anesthesia, which reduces hemodynamic fluctuation and maintains the ideal depth of anesthesia during the operation (Witkowska et al., 2012; Koo et al., 2012; Yeganeh et al., 2013; Dussaussoy et al., 2014). The availability of TCI of propofol and remifentanil hydrochloride in burn patients was confirmed in our pre-application study (Guo et al., 2012).

The clinical application of the bispectral index (BIS) was approved by the U.S. Food and Drug Administration in 1996. BIS reflects the sedation depth of patients receiving surgery, increases the safety of anesthesia, and reduces the dosage of intravenous anesthetics (López Navarro et al., 2004; Han et al., 2005). However, currently, anesthetic depth in patients with severe burns is still primarily evaluated based on clinical signs, which lacks objective and valid judgment indices. Thus, this study aimed to explore the feasibility and effectiveness of the use of BIS for monitoring the intraoperative depth of anesthesia with propofol and remifentanil TCI in patients with severe burns.

\section{MATERIAL AND METHODS}

We recruited 80 patients with severe burns who underwent elective escharectomy $(<1$ week) at the First Affiliated Hospital of People's Liberation Army General Hospital between December 2011 and December 2012.

\section{Patients}

All patients [classes II to III of the American Society of Anesthesiologists (ASA) classification] had total burn surface areas ranging from 31 to $50 \%$ or third-degree burn injuries ranging from 11 to $20 \%$. The ages of the patients ranged from 18 to 65 years, and their weights 
ranged from 40 to $90 \mathrm{~kg}$. The patients were randomly assigned to BIS (A) and control (B) groups (20 cases in each group) according to the random-number table method. Patients were excluded if they had drug allergies, apparent heart, lung, liver or kidney dysfunction before the operation; and who were seriously overweight [body mass index (BMI) $>30 \mathrm{~kg} / \mathrm{m}^{2}$ ]. No significant differences were found between the 2 groups regarding gender, age, BMI, height, total body surface area, full-thickness burn surface area, days from injury to surgery, and operation time ( $P>0.05$, Table 1). This study was conducted in accordance with the Declaration of Helsinki and approved by the Ethics Committee of First Affiliated Hospital of General Hospital of PLA. Written informed consent was obtained from the participants. If patients exhibited a language communication or written language problem before the operation, we consulted with the patients while they were conscious, and simultaneously obtained informed consent from them or from a legal representative.

\begin{tabular}{|c|c|c|c|c|c|c|c|c|c|}
\hline \multirow[t]{2}{*}{ Group } & \multicolumn{2}{|c|}{ Gender (cases) } & \multirow[t]{2}{*}{ Age (year) } & \multirow[t]{2}{*}{ Weight (kg) } & \multirow[t]{2}{*}{ Height (cm) } & \multirow[t]{2}{*}{ ST (min) } & \multirow[t]{2}{*}{ TBSA } & \multirow[t]{2}{*}{ FTBSA } & \multirow[t]{2}{*}{ Days } \\
\hline & Male & Female & & & & & & & \\
\hline Group A & 26 & 14 & $42.50 \pm 13.33$ & $63.65 \pm 9.29$ & $167.75 \pm 6.11$ & $178.00 \pm 36.40$ & $0.39 \pm 0.08$ & $0.15 \pm 0.03$ & $2.78 \pm 0.64$ \\
\hline Group B & 28 & 12 & $39.95 \pm 14.70$ & $64.80 \pm 10.80$ & $166.55 \pm 6.50$ & $183.00 \pm 33.97$ & $0.42 \pm 0.07$ & $0.15 \pm 0.02$ & $2.83 \pm 0.69$ \\
\hline
\end{tabular}

Group A = BIS group; Group B = Control group; “.” = no statistical value; ST = surgery time; TBSA = total burn surface area; FTBSA = full thickness burn surface area; Days = days from injury to surgery.

\section{Anesthesia}

All patients were anesthetized by a the same anesthesiologist. Upon the arrival of the patients at the operating theater, an intravenous line was placed and Ringer's solution $(10 \mathrm{~mL} /$ $\mathrm{kg}$ ) was administered until measurements were initiated. Non-invasive blood pressure and invasive arterial pressure were measured, and ECG and pulse oximeter monitors were attached. Vital signs were monitored routinely by using a BIS monitor (A-2000 type; Aspect Medical Systems Inc., USA). The TCI system is consisted of a Minto remifentanil pharmacokinetic model (Alaris @ PK, ALARIS Medical, UK) and a Marsh propofol pharmacokinetic model (Graseby3500, Smith Medical, U.S.A) (Witkowska et al., 2012). The initial target effect-site concentration of remifentanil was $2.5 \mathrm{ng} / \mathrm{mL}$. At 2 min after infusion, propofol was administered via TCI. Both groups received propofol at an initial target plasma concentration of $2.0 \mu \mathrm{g} / \mathrm{mL}$. The plasma concentration was increased by $0.5 \mu \mathrm{g} / \mathrm{mL}$ per minute until the patients lost consciousness. During anesthesia induction, $2 \mathrm{mg}$ of dopamine was administered if the mean arterial pressure (MAP) decreased by more than $20 \%$. The patients received $0.15 \mathrm{mg} / \mathrm{kg}$ cisatracurium besylate and underwent transoral tracheal intubation for mechanical ventilation. The oxygen flow rate was $2 \mathrm{~L} /$ min, and the tidal volume was $10 \mathrm{~mL} / \mathrm{kg}$. The respiratory rate was adjusted to maintain the endtidal pressure of carbon dioxide between 35 and $45 \mathrm{mmHg}(1 \mathrm{mmHg}=0.133 \mathrm{kPa})$. We administered cisatracurium besylate to the patients to maintain muscle relaxation.

At the preparation stage of surgery, the target effect-site concentration of remifentanil was decreased by 0.5 to $1 \mathrm{ng} / \mathrm{mL}$ in both groups. Before the operation, the remifentanil concentration was returned to the induction level. In the BIS group, the plasma concentration of propofol was adjusted according to the BIS, and was maintained between 40 and 60 . In the control group, the plasma concentration of propofol was adjusted according to blood pressure, heart rate (HR), and body movement. The plasma concentrations of propofol were adjusted 
in $0.5 \mu \mathrm{g} / \mathrm{mL}$ increments, separated by intervals of more than $1 \mathrm{~min}$. According to HR and blood pressure, the target effect-site concentration and infusion rate of remifentanil were also adjusted $(0.5 \mathrm{ng} / \mathrm{mL}$ per adjustment). Cardiovascular agents such as ephedrine, dopamine, atropine, esmolol, and urapidil were administered when necessary.

After skin suturing, propofol and remifentanil were withdrawn. Tramadol at $1 \mathrm{mg} / \mathrm{kg}$ was administered $30 \mathrm{~min}$ before the end of the operation. The patients recovered consciousness after the operation. Sputum suction and extubation were performed when the indications for extubation were satisfied.

\section{Data collection}

MAP, HR, and BIS of the patients were monitored during the entire course of the operation. MAP and HR were recorded at the following time points: upon entering the operating room; at the time of unconsciousness; 2 min after intubation; before the operation; 2, 15, and $30 \mathrm{~min}$ from the start of the operation; after the operation; at the time of eye opening after the patient heard his/her name called; and the time to reach an Aldrete score of 9 points. The target concentrations of remifentanil and propofol during anesthesia maintenance, the time to eye opening, the time to reach an Aldrete score of 9 points from drug withdrawal, and the BIS values at the aforementioned time points were also recorded. All patients were queried about intraoperative awareness after the operation.

\section{Data analysis}

Data were analyzed by using the SPSS11.0 software (SPSS, Chicago, IL). All data measured are reported as means \pm standard deviation. The $t$-tests were used to make comparisons between groups. ANOVA was used to compare results within the same group, and chi-square tests were used for categorical data. The prediction probability $\left(P_{k}\right)$ was used to evaluate the prediction efficacy of BIS, MAP, and HR for the changes in consciousness during recovery. Spearman's correlation analysis was performed to determine the correlations between these indices and the changes in consciousness during recovery. Differences with $\mathrm{P}<$ 0.05 were considered to be statistically significant.

\section{RESULTS}

\section{Perianesthesia drug target concentrations and recovery of consciousness}

Compared with group B, the patients in group A had significantly lower target concentrations of remifentanil and propofol during anesthesia maintenance and significantly shorter times to eye opening upon hearing his/her name called and to reaching an Aldrete score of 9 points from drug withdrawal $(\mathrm{P}<0.01$; Table 2$)$.

\section{Perioperative MAP and HR changes}

In both groups, the MAP and HR values at different time points after loss of consciousness were significantly lower compared with the baseline values (values upon entering the operating room; $\mathrm{P}<0.05$ ), with the exception of $2 \mathrm{~min}$ after intubation. These values 
were stably maintained during the operation. During loss of consciousness, group A showed a slower decrease in MAP values than group B, which suggests that the hemodynamics were more stable under BIS monitoring at this time point. No significant differences in HR were observed. At the other time points, neither group exhibited significant differences in MAP or HR (Table 3). According to the postoperative follow-ups, all patients in both groups were unaware of the events during the operation.

Table 2. Comparison of the target concentration of drug and awake during anesthetic maintenance (mean \pm standard deviation).

\begin{tabular}{lcccc}
\hline Group & $\begin{array}{c}\text { Effect-site concentration } \\
\text { of remifentanil }(\mathrm{ng} / \mathrm{mL})\end{array}$ & $\begin{array}{c}\text { Plasma concentration } \\
\text { of propofol }(\mu \mathrm{g} / \mathrm{mL})\end{array}$ & $\begin{array}{c}\text { Time for eyes } \\
\text { opening }(\mathrm{min})\end{array}$ & $\begin{array}{c}\text { Aldrete score } \\
9 \text { points }(\mathrm{min})\end{array}$ \\
\hline Group A & $2.12 \pm 0.35$ & $2.54 \pm 0.22$ & $7.90 \pm 0.58$ & $9.15 \pm 0.69$ \\
Group B & $2.50 \pm 0.21^{1}$ & $2.86 \pm 0.31^{1}$ & $8.35 \pm 0.66^{1}$ & $11.13 \pm 0.96^{1}$ \\
\hline
\end{tabular}

Group A = BIS group; Group B $=$ Control group. ${ }^{1} \mathrm{P}<0.01$ compared with group A.

Table 3. MAP and HR changes during anesthetic maintenance (mean \pm standard deviation).

\begin{tabular}{lllll}
\hline Time & \multicolumn{2}{c}{ MAP } & & HR \\
\cline { 2 - 3 } & Group A & Group B & Group A & Group B \\
\hline Enter the operation room & $76.08 \pm 7.34$ & $74.45 \pm 7.43$ & $79.80 \pm 7.46$ & $78.55 \pm 5.82$ \\
Loss of consciousness & $65.73 \pm 7.69^{2}$ & $62.20 \pm 4.23^{1,2}$ & $64.05 \pm 7.05^{2}$ & $65.60 \pm 4.17^{2}$ \\
2 min after intubation & $73.95 \pm 6.51$ & $73.63 \pm 7.55$ & $72.48 \pm 6.97^{2}$ & $72.25 \pm 6.23^{2}$ \\
Before surgery & $71.35 \pm 6.16^{2}$ & $71.15 \pm 6.79^{2}$ & $60.10 \pm 6.08^{2}$ & $61.65 \pm 4.59^{2}$ \\
2 min after the start of surgery & $72.20 \pm 4.05^{2}$ & $70.83 \pm 5.06^{2}$ & $74.38 \pm 7.16^{2}$ & $72.40 \pm 6.76^{2}$ \\
15 min after the start of surgery & $72.80 \pm 2.76^{2}$ & $70.03 \pm 3.83^{2}$ & $73.55 \pm 6.94^{2}$ & $73.48 \pm 6.05^{2}$ \\
30 min after the start of surgery & $69.50 \pm 3.80^{2}$ & $70.58 \pm 3.72^{2}$ & $72.00 \pm 5.24^{2}$ & $70.98 \pm 4.47^{2}$ \\
Surgery end & $70.48 \pm 4.88^{2}$ & $70.15 \pm 4.20^{2}$ & $65.95 \pm 6.52^{2}$ & $66.90 \pm 6.19^{2}$ \\
\hline
\end{tabular}

Group A = BIS group; Group B = Control group; Compared with group A ${ }^{1} \mathrm{P}<0.05$; compared with the time of Enter the operation room ${ }^{2} \mathrm{P}<0.05$.

\section{BIS, MAP, HR, and the changes in consciousness during recovery from anesthesia}

In group A patients, the $P_{k}$ values of BIS, in contrast to those of MAP and HR, for the times from the end of the operation to eye opening upon hearing his/her name called $\left(T_{1}-T_{2}\right)$ and to orientation restoration $\left(\mathrm{T}_{1}-\mathrm{T}_{3}\right)$ during consciousness recovery were significantly higher than 0.5 ( $\mathrm{P}<0.05$; Table 4). The Spearman's correlation coefficient of BIS with changes in consciousness was $0.647(\mathrm{P}<0.05)$, whereas those of MAP and HR were 0.326 and 0.241 , respectively.

Table 4. BIS, MAP, HR predicted awareness of changes in the patient recovery period $P_{k}$ and correlation coefficient (Spearman) (mean \pm standard deviation).

\begin{tabular}{lccr}
\hline Outcome measures & \multicolumn{3}{c}{} \\
\cline { 2 - 3 } & $\mathrm{T}_{1}-\mathrm{T}_{2}$ & $P_{k}$ & \multirow{2}{*}{ Spearman } \\
\hline BIS & $0.87 \pm 0.03$ & $0.89 \pm 0.02$ & $0.647^{1}$ \\
MAP & $0.59 \pm 0.08$ & $0.68 \pm 0.02$ & 0.326 \\
HR & $0.51 \pm 0.07$ & $0.56 \pm 0.04$ & 0.241 \\
\hline
\end{tabular}

$\mathrm{BIS}=$ bispectral index; $\mathrm{MAP}=$ mean artery pressure $\mathrm{HR}=$ heart rate; $\mathrm{T}_{1}-\mathrm{T}_{2}=$ the end of surgery to the call of the eyes open; $T_{1}-T_{3}=$ the end of surgery to the orientation recovery (an Aldrete score of 9 points); BIS indicates the monitoring parameter in BIS group; mean arterial pressure and heart rate indicate the monitoring parameter in control group; Spearman indicates the correlation of $\mathrm{T}_{1}-\mathrm{T}_{3}$ and relevant $P_{k}$ value in each test item. ${ }^{1} \mathrm{P}<0.05$ compared with $P_{k}=0.5$. 


\section{DISCUSSION}

In this study, BIS was used to monitor anesthetic depth in patients with severe burns who underwent elective escharectomy $(<1$ week) during general anesthesia via TCI of remifentanil and propofol. Compared to the values at the end of the operation, the BIS, HR, and MAP values were significantly changed at the time of eye opening and the time of an Aldrete score of 9 points. Of these, the change in BIS in the BIS group was significantly correlated with the change in consciousness awakening, and the correlation was significantly higher than that of the conventional hemodynamic index in the control group. Therefore, we propose that changes in BIS could be used to predict changes in consciousness during recovery from general anesthesia. Compared with group B, the patients in group A had significantly lower target concentrations of remifentanil and propofol during anesthesia maintenance and considerably shorter times to eye opening upon hearing his/her name called and to reaching an Aldrete score of 9 points.

The target-controlled concentrations of remifentanil and propofol are high in burn patients during anesthesia (López Navarro et al., 2004; Yamashita et al., 2010). In this study, patients with burns of ASA grades II to III were recruited. TCI with fentanyl and propofol has been used to anesthetize these patients because they have supplemented blood volumes and lack sepsis (Koo et al., 2012). Because patients with severe burns have unstable hemodynamics, their anesthetic depth is unlikely to be evaluated accurately merely on the basis of basic vital signs, such as blood pressure and HR. As a frequently used index for monitoring anesthetic depth in clinical practice, BIS satisfactorily correlates with cerebral cortex-acting drugs, such as propofol, midazolam, and sevoflurane, and can reliably evaluate the anesthetic depth and predict the changes in consciousness during and after an operation (Albertin et al., 2005; Bottros et al., 2011; Fan et al., 2013). The results of this study showed that BIS was highly correlated with the target-controlled concentration of propofol in burn patients during surgery. BIS effectively reflected anesthetic depth in these patients. In addition, a negative correlation was detected between BIS and the target-controlled concentration of propofol. These findings are consistent with the results previously reported by other authors (Klopman and Sebel, 2011; Kaskinoro et al., 2011; Orhon et al., 2013).

TCI is a novel, individualized anesthesia method (Sahinovic et al., 2010). The results of this study showed that the MAP and HR values were stably maintained in both groups during the operation, although the values at the time of loss of consciousness were lower than those upon entering the operating room during the perioperative period. Significant differences in MAP values between the 2 groups at the time of loss of consciousness were observed. These differences were presumably due to more precise control under BIS in the experimental group. Perioperative hemodynamics was stable in both groups, which indicated that the effects of this anesthesia method on MAP and HR of patients are controllable.

$P_{k}$, a prediction probability concept, was first proposed to compare the prediction efficiencies of different anesthetic depth indices by Smith et al. (1996). $P_{k}$, which ranges from 0 to 1 , is subjected to nonparametric statistical methods and is not influenced by data distribution patterns and sample size. A $P_{k}$ value of 1 indicates that the prediction accuracy of the related monitoring index for anesthetic depth is $100 \%$, whereas a value of 0.5 indicates that the prediction accuracy is $50 \%$. Thus, a $P_{k}$ value of 0.5 indicates that the related index has no prediction value. A larger $P_{k}$ value denotes higher prediction accuracy. In this study, the prediction accuracies of BIS, MAP, and HR for eye opening and consciousness recovery of patients (an Aldrete score of 9 points) were evaluated. The results showed that the $P_{k}$ values of BIS, MAP, 
and HR were significantly higher than 0.5 , which indicated that BIS reliably reflects the changes in consciousness of patients during recovery from anesthesia (Ouyang and Zhang, 2006).

In this study, BIS was used to monitor anesthetic depth in patients with severe burns, and it successfully prevented the occurrence of adverse events, such as prolonged recovery time. BIS can accurately predict the changes in the consciousness levels of patients during recovery from general anesthesia (Haller et al., 2009). This study provides a clinical basis for the reasonable application of BIS in monitoring anesthetic depth in patients with severe burns.

\section{Conflicts of interest}

The authors declare no conflict of interest.

\section{REFERENCES}

Albertin A, Casati A, Federica L, Roberto V, et al. (2005). The effect-site concentration of remifentanil blunting cardiovascular responses to tracheal intubation and skin incision during bispectral index-guided propofol anesthesia. Anesth. Analg. 101: 125-130.

Bottros MM, Palanca BJ, Mashour GA, Patel A, et al. (2011). Estimation of the bispectral index by anesthesiologists: an inverse turing test. Anesthesiology 114: 1093-1101.

Brunette KE, Anderson BJ, Thomas J, Wiesner L, et al. (2011). Exploring the pharmacokinetics of oral ketamine in children undergoing burns procedures. Paediatr. Anaesth. 21: 653-662.

Dussaussoy C, Peres M, Jaoul V, Liu N, et al. (2014). Automated titration of propofol and remifentanil decreases the anesthesiologist's workload during vascular or thoracic surgery: a randomized prospective study. J. Clin. Monit. Comput. 28: 35-40.

Fan TW, Ti LK and Islam I (2013). Comparison of dexmedetomidine and midazolam for conscious sedation in dental surgery monitored by bispectral index. Br. J. Oral. Maxillofac. Surg. 51: 428-433.

Guo ZG, Wang XY, Lu XL, Su XJ et al. (2012). Application of Narcotrend-assisted anesthesia in-depth monitor during escharectomy and skin transplantation in burn patients with target-controlled infusion of remifentanil hydrochloride and propofol. Zhonghua shao shang za zhi Chin. J. burns 28: 178-182.

Haller G, Stoelwinder J, Myles PS and McNeil J (2009). Quality and safety indicators in anesthesia: a systematic review. Anesthesiology 110: 1158-1175.

Han TH, Lee JH, Kwak IS, Kil HY, et al. (2005). The relationship between bispectral index and targeted propofol concentration is biphasic in patients with major burns. Acta Anaesthesiol. Scand. 49: 85-91.

Han TH, Greenblatt DJ and Martyn JA (2009). Propofol clearance and volume of distribution are increased in patients with major burns. J. Clin. Pharmacol. 49: 768-772.

Kaneda K and Han TH (2009). Comparative population pharmacokinetics of fentanyl using non-linear mixed effect modeling: burns vs. non-burns. Burns 35: 790-797.

Kaskinoro K, Maksimow A, Långsjö J and Struys MM (2011). Wide inter-individual variability of bispectral index and spectral entropy at loss of consciousness during increasing concentrations of dexmedetomidine, propofol, and sevoflurane. Br. J. Anaesth. 107: 573-580.

Klopman MA and Sebel PS (2011). Cost-effectiveness of bispectral index monitoring. Curr. Opin. Anaesthesiol. 24: 177-181.

Koo BN, Lee JR, Noh GJ, Lee JH, et al. (2012). A pharmacodynamic analysis of factors affecting recovery from anesthesia with propofol-remifentanil target controlled infusion. Acta. Pharmacol. Sin. 33: 1080-1084.

López Navarro AM, Peiró C, Matoses S, Silvestre M, et al. (2004). General anesthesia by infusion of remifentanil for debridement and grafting of large burns in 3 pediatric patients. Rev. Esp. Anestesiol. Reanim. 51: 47-50.

Orhon ZN, Devrim S, Celik M, Dogan Y, et al. (2013). Comparison of recovery profiles of propofol and sevoflurane anesthesia with bispectral index monitoring in percutaneous nephrolithotomy. Korean J. Anesthesiol. 64: 223-228.

Ouyang MW and Zhang H (2006). Comparison of unconsciousness prediction probability with approximate entropy and bispectral index during sedation. Nan Fang Yi Ke Da Xue Xue Bao 26: 287-289.

Sahinovic MM, Absalom AR and Struys MM (2010). Administration and monitoring of intravenous anesthetics. Curr. Opin. Anaesthesiol. 23: 734-740.

Smith WD, Dutton RC and Smith NT (1996). Measuring the performance of anesthetic depth of indicators. Anesthesiology 84: 38-51. 
Witkowska M, Karwacki Z, Rzaska M, Niewiadomski S, et al. (2012). Comparison of target controlled infusion and total intravenous anaesthesia with propofol and remifentanil for lumbar microdiscectomy. Anaesthesiol Intensive Ther. 44: $138-144$

Yamashita S, Kaneda K, Han TH (2010). Population pharmacokinetics of a propofol bolus administered in patients with major burns. Burns 36:1215-1221.

Yeganeh N, Roshani B, Latifi H, Almasi A (2013). Comparison of target-controlled infusion of sufentanil and remifentanil in blunting hemodynamic response to tracheal intubation. J. Inj. Violence Res. 5: 101-107. 